\title{
ON PROJECTIONS WITH NORM 1-AN EXAMPLE
}

\author{
JORAM LINDENSTRAUSS ${ }^{1}$
}

Let $X$ be a Banach space. It is a well-known result that if for every Banach space $Y \supset X$ with $\operatorname{dim} Y / X=1$ there is a projection with norm 1 from $Y$ onto $X$, then the same holds for every $Y \supset X$ without any restriction on $Y / X$ (see, for example, [3] and the references given there). In [2] we proved that if for every $Y \supset X$ and every $\epsilon>0$ there is a projection with norm $\leqq 1+\epsilon$ from $Y$ onto $X$ then there is also a projection with norm 1 from every $Y \supset X$ onto $X$.

In view of these results the following questions naturally arise (cf. also [3, problem 6$]$ ):

1. Let $Z \supset X$ be Banach spaces. Suppose that for every $Y$ with $Z \supset Y \supset X$ and $\operatorname{dim} Y / X=1$ there is a projection with norm 1 from $Y$ onto $X$. Does there exist a projection with norm 1 from $Z$ onto $X$ ?

2. Let $Z \supset X$ be Banach spaces. Suppose that for every $\epsilon>0$ there is a projection with norm $\leqq 1+\epsilon$ from $Z$ onto $X$. Does there exist a projection with norm 1 from $Z$ onto $X$ ?

The answer to both questions is negative. This can be shown by rather simple examples. The purpose of this note is to show that even if the assumptions of both 1 and 2 hold, there may be no projection with norm 1 from $Z$ onto $X$. We shall prove the following.

Theorem. There exist Banach spaces $Z \supset X$ with $\operatorname{dim} Z / X=2$ satisfying:

(i) There is no projection with norm 1 from $Z$ onto $X$.

(ii) For every $\epsilon>0$ there is a projection with norm $\leqq 1+\epsilon$ from $Z$ onto $X$.

(iii) For every $Y$ with $Z \supset Y \supset X$ and $\operatorname{dim} Y / X=1$ there is a projection with norm 1 from $Y$ onto $X$.

Before constructing the spaces $Z$ and $X$ we introduce some notations. Let $K$ be the compact metric space of all the ordinals $\leqq \omega^{2}$ in the order topology. ${ }^{2}$ Let $K_{m}, m=1,2, \cdots$, be the subsets of $K$ defined by

$$
K_{m}=\{\alpha ;(m-1) \omega<\alpha \leqq m \omega\} .
$$

Clearly $K-\left\{\omega^{2}\right\}=\cup_{m=1}^{\infty} K_{m} \cdot{ }^{3}$ Let $N$ denote the set of positive inte-

Received by the editors February 23, 1963.

1 Research supported by NSF Grant G-25222.

$2 \omega$ denotes, as usual, the ordinal number of the well-ordered set of the integers.

$3\left\{\omega^{2}\right\}$ denotes the set consisting of the single point $\omega^{2}$. We do not consider 0 here as an ordinal number. 
gers. Let $h(\alpha)$ be the function on $K$ defined by

(2) $\quad h(\alpha)=\left\{\begin{aligned} 1 & \text { if } \alpha=m \omega+2 j-1, m=0,1,2, \cdots, j=1,2, \cdots, \\ -1 & \text { otherwise. }\end{aligned}\right.$

Further let $f_{n}, n \in N$, be a sequence of continuous functions on $K$ defined by

$$
f_{n}(\alpha)=\left\{\begin{aligned}
-1 & \text { if } \alpha \in K_{2 m-1}, m=1,2, \cdots, n, \\
1 & \text { otherwise. }
\end{aligned}\right.
$$

The functions $f_{n}$ converge (pointwise) as $n \rightarrow \infty$ to the function $f$ defined by

$$
f(\alpha)=\left\{\begin{aligned}
-1 & \text { if } \alpha \in K_{2 m-1}, m=1,2, \cdots, \\
1 & \text { otherwise. }
\end{aligned}\right.
$$

We are now ready to define the spaces $Z$ and $X$. All the spaces will be over the real field. Let $V$ be the space of all the bounded realvalued functions on the (abstract) set $K \times N$, with the usual vector operations and the sup as norm. As $X$ we take the subspace of $V$ consisting of all the functions $v$ satisfying $v(\alpha, n)=v(\alpha, 1)$ for every $\alpha \in K$ and $n \in N$, and $v(\alpha, 1) \in C(K) .{ }^{4}$ The mapping $T_{0}$ from $X$ onto $C(K)$ defined by

$$
T_{0} x(\alpha)=x(\alpha, 1), \quad x \in X, \alpha \in K,
$$

is clearly an isometry. As $Z$ we take the subspace of $V$ spanned by $X$ and the functions

$$
\begin{aligned}
& z_{1}(\alpha, n)=f_{n}(\alpha), \quad \alpha \in K, n \in N, \text { and } \\
& z_{2}(\alpha, n)=h(\alpha) / n, \quad \alpha \in K, n \in N .
\end{aligned}
$$

Before turning to the proof that (i)-(iii) hold we remark that there is a projection with norm $\leqq \lambda$ from $Z$ [resp. from a subspace $Y$ of $Z$ containing $X$ ] onto $X$ if and only if there is an extension of $T_{0}$ from $Z$ [resp. $Y$ ] onto $C(K)$ with norm $\leqq \lambda$. Further, we recall that there is a biunique correspondence between operators $T$ from a Banach space $U$ into $C(K)$ and $w^{*}$ continuous mappings $F$ from $K$ to $U^{*}$. This correspondence is given by the equation $T u(\alpha)=F(\alpha) u, \alpha \in K$, $u \in U$. Moreover, $\|T\|=\sup _{\alpha}\|F(\alpha)\|$ (cf. [1, p. 492]). The function $F_{0}$ from $K$ to $X^{*}$ corresponding to the operator $T_{0}$ defined in (5) is given by

- $C(K)$ denotes the Banach space of all the continuous real-valued functions on $K$ with the sup norm. 
(8) $\quad F_{0}(\alpha) x=x(\alpha, 1)=x(\alpha, n), \quad x \in X, n \in N, \alpha \in K$.

Proof of (i). By the preceding remarks and the Hahn-Banach Theorem we have to show that there is no function $F$ from $K$ to $V^{*}$ having the following properties:

$$
\begin{aligned}
F(\alpha)_{\mid X} & =F_{0}(\alpha),{ }^{5} & & \alpha \in K, \\
\|F(\alpha)\| & =1, & & \alpha \in K, \\
F(\alpha) z_{i} & \in C(K), & & i=1,2 .
\end{aligned}
$$

Suppose there is such an $F$. By the well-known representation of $V^{*}$ we may consider each $F(\alpha)$ as a finitely additive measure on $K \times N$. Let $\alpha$ be an isolated point of $K$. The characteristic function $\chi_{\alpha}$ of the set $\{\alpha\} \times N$ belongs to $X$ and $F_{0}(\alpha) \chi_{\alpha}=1$. Hence by (9) and (10) $F(\alpha)$ is a positive measure with norm 1 vanishing outside $\{\alpha\} \times N$. Since $\lim _{n \rightarrow \infty} z(\alpha, n)$ exists for every $z \in Z$ and $\alpha \in K$ it follows that for isolated $\alpha \in K$ there are non-negative numbers $a_{\alpha, n}$, $n \in N$, and $a_{\alpha, \infty}$ satisfying

$$
\begin{gathered}
\sum_{n=1}^{\infty} a_{\alpha, n}+a_{\alpha, \infty}=1, \text { and } \\
F(\alpha) z=\sum_{n=1}^{\infty} a_{\alpha, n} z(\alpha, n)+a_{\alpha, \infty} \lim _{n \rightarrow \infty} z(\alpha, n) \quad z \in Z .
\end{gathered}
$$

By (7) we obtain that $F(\alpha) z_{2}=h(\alpha) \sum_{n=1}^{\infty} a_{\alpha, n} / n$. By the definition of $h$ and by (11) it follows that as $\alpha$ tends to $m \omega(m=1,2, \cdots)$, $\sum a_{\alpha, n} / n$ tends to 0 , and since the $a_{\alpha, n}$ are non-negative we obtain

$$
\lim _{\alpha \rightarrow m \omega} a_{\alpha, n}=0, \quad n, m=1,2, \cdots \text {. }
$$

For isolated $\alpha \in K_{2 m}, m=1,2, \cdots$, we obtain by (3), (4), (6), (12) and (13) that $F(\alpha) z_{1}=1$. Hence by (11)

$$
F\left(\omega^{2}\right) z_{1}=1 .
$$

For isolated $\alpha \in K_{2 m+1}, m=1,2, \cdots$, we obtain similarly that

$$
F(\alpha) z_{1}=2\left(a_{\alpha, 1}+a_{\alpha, 2}+\cdots+a_{\alpha, m}\right)-1,
$$

and hence by (11) and (14), $F((2 m+1) \omega) z_{1}=-1$. But this contradicts (11) and (15).

Proof of (ii). Let $T_{n}$ be the linear operator from $Z$ to $C(K)$ defined by

${ }^{6} F(\alpha)_{\mathrm{IX}}$ denotes the restriction of $F(\alpha)$ to $X$. 


$$
T_{n}\left(x+\lambda z_{1}+\mu z_{2}\right)=x(\alpha, n)+\lambda z_{1}(\alpha, n), \quad x \in X, \lambda, \mu \text { scalars. }
$$

Clearly $T_{n \mid X}=T_{0}$ for every $n \in N$. We estimate the norm of $T_{n}$. There exists an $M<\infty$ such that $|\mu|<M\left\|x+\lambda z_{1}+\mu z_{2}\right\|$ for every $x, \lambda$ and $\mu$. Hence

$$
\begin{aligned}
\left\|T_{n}\left(x+\lambda z_{1}+\mu z_{2}\right)\right\| & \leqq \sup _{\alpha}\left|x(\alpha, n)+\lambda z_{1}(\alpha, n)+\mu z_{2}(\alpha, n)\right|+|\mu| / n \\
& \leqq(1+M / n)\left\|x+\lambda z_{1}+\mu z_{2}\right\| .
\end{aligned}
$$

This proves (ii).

Proof of (iii). If $Y$ is the subspace of $Z$ spanned by $X$ and $z_{2}$ then the operator $T$ from $Y$ to $C(K)$ defined by $T\left(x+\lambda z_{2}\right)=T_{0}(x)$ is a norm preserving extension of $T_{0}$. Hence we may assume that $Y$ is the subspace of $Z$ spanned by $X$ and $y=z_{1}+\mu z_{2}$. By reversing the argument used in the proof of (i) it follows that it is sufficient to show that for isolated $\alpha \in K$ there exist non-negative $a_{\alpha, n}, n \in N$, and $a_{\alpha, \infty}$ satisfying (12) such that the function

$$
g(\alpha)=\sum_{n=1}^{\infty} a_{\alpha, n} f_{n}(\alpha)+\mu h(\alpha) \sum_{n=1}^{\infty} a_{\alpha, n} / n+a_{\alpha, \infty} f(\alpha)
$$

has a continuous extension to the whole of $K$. We choose the $a_{\alpha, n}$ and $a_{\alpha, \infty}$ as follows. For $\alpha \in K_{2 m}, m=1,2, \cdots$ and for $\alpha \in K_{2 m+1}$ with $2 m \leqq|\mu|$ we take $a_{\alpha, \infty}=1$ and $a_{\alpha, n}=0, n \in N$. For $\alpha \in K_{2 m+1}$ with $2 m>|\mu|$ we take $a_{\alpha, m}=1$, and $a_{\alpha, n}=a_{\alpha, \infty}=0, n \neq m$ if $\operatorname{sgn} \mu h(\alpha)=-1,{ }^{6}$ while if $\operatorname{sgn} \mu h(\alpha)=1$ we take $a_{\alpha, m}=(2 m-|\mu|) /(2 m+|\mu|), a_{\alpha, \infty}$ $=1-a_{\alpha, m}$ and $a_{\alpha, n}=0, n \neq m$. With this choice of the $a_{\alpha, n}$ and $a_{\alpha, \infty}$ we have, for isolated $\alpha$,

$$
g(\alpha)= \begin{cases}-1 & \text { if } \alpha \in K_{2 m+1} \text { with } 2 m \leqq|\mu|, \\ 1 & \text { if } \alpha \in K_{2 m}, m=1,2, \cdots, \\ 1-|\mu| / m & \text { if } \alpha \in K_{2 m+1} \text { with } 2 \mathrm{~m}>|\mu| .\end{cases}
$$

This $g$ has, clearly, a continuous extension to $K$, and this concludes the proof of assertion (iii).

\section{REFERENCES}

1. N. Dunford and J. Schwartz, Linear operators. I, Interscience, New York, 1958.

2. J. Lindenstrauss, The extension of compact operators. III, Technical note no. 32, Jerusalem, 1962, Trans. Amer. Math. Soc. (to appear).

3. L. Nachbin, Some problems in extending and lifting linear transformations, Proc. Internat. Sympos. Linear Spaces, pp. 340-350, Jerusalem, 1961.

YaLE University

- We define $\operatorname{sgn} t=1$ if $t \geqq 0$ and $\operatorname{sgn} t=-1$ if $t<0$ 